\title{
Case Report Primary Cardiac Fibroma in an Adult
}

\author{
Samuel H. Cho, ${ }^{1}$ Timothy Fritz, ${ }^{2}$ Lynn J. Cronin, ${ }^{2}$ and Stephen D. Cohle ${ }^{3}$ \\ ${ }^{1}$ Michigan State University College of Human Medicine, Grand Rapids, MI, USA \\ ${ }^{2}$ Spectrum Health Frederik Meijer Heart \& Vascular Institute, Grand Rapids, MI, USA \\ ${ }^{3}$ Spectrum Health Department of Pathology, Grand Rapids, MI, USA \\ Correspondence should be addressed to Samuel H. Cho; sam77cho@gmail.com
}

Received 7 August 2015; Accepted 6 September 2015

Academic Editor: Ramazan Akdemir

Copyright (C) 2015 Samuel H. Cho et al. This is an open access article distributed under the Creative Commons Attribution License, which permits unrestricted use, distribution, and reproduction in any medium, provided the original work is properly cited.

Cardiac fibromas are benign primary tumors composed of connective tissue and fibroblasts. These uncommon tumors are primarily found in the pediatric population, and their prevalence among the adult population is exceedingly rare. We report a case of an adult with nonspecific symptoms, who was subsequently found to have a solitary mass located in the left ventricle. This case highlights an unusual finding in an adult who through various imaging modalities, surgical excision, and immunohistological analysis was found to have a cardiac fibroma.

\section{Introduction}

Cardiac fibromas are benign primary tumors composed of connective tissue and fibroblasts. These uncommon tumors are primarily found in the pediatric population [1], and their prevalence among the adult population is exceedingly rare. We present a case of an adult with nonspecific symptoms, who was subsequently discovered to have a solitary mass located in the left ventricle suggestive of a cardiac fibroma.

\section{Case Presentation}

A 60-year-old woman presented with an acute onset of fatigue, shortness of breath, chest pain, and palpitations, following an episode of bronchitis. The patient also had a 7pound weight gain over the past 1-2 weeks. She was found to have moderate pericardial effusion, and during preliminary imaging for echocardiographic guided pericardiocentesis a mass was noted on the epicardial surface of the left ventricle. The pericardiocentesis was deferred, and the patient underwent further diagnostic imaging.

An echocardiogram conclusion was a large, well-circumscribed epicardial mass on the left ventricular surface of the heart, not consistent with a left ventricular pseudoaneurysm. The mass was solid, and it appeared to sit on top of the normal myocardium. Cardiac MRI with and without contrast showed an approximate $4 \mathrm{~cm}$ broad based attachment to the inferolateral myocardium of the left ventricle apex (Figures $1(\mathrm{a})$ and $1(\mathrm{~b})$ ). The mass was $\mathrm{T} 1$ isointense to muscle with areas of T2 hypointensity, suggestive of fibrosis [2]. Noncontrast chest CT revealed a low attenuation mass with prominent calcification at the left ventricular apex with moderately sized pericardial effusion (Figures 1(c) and 1(d)).

Complete surgical resection was carried out under cardiopulmonary bypass. The operative finding was $5.5 \times 4.0 \times$ $3.5 \mathrm{~cm}$ tan-brown colored mass at the posterior lateral portion of the heart near the apex (Figure 2). The tumor was found to be in close proximity to the left anterior descending artery. The edge of the tumor was discerned and was not invading into the myocardium. Closure was made with a patch, and postprocedure transesophageal echocardiogram demonstrated well-preserved left ventricular and right ventricular function.

Sectioning revealed tan-white, rubbery, whorled, calcified cut surfaces (Figure 2(b)). Neither hemorrhage nor necrosis was present and the mass was sent for histopathological examination. Microscopically, a trichrome stain highlighted a sharp demarcation between the fibroma (blue) and normal myocardium (red) (Figure 3(a)). At higher power, the fibroma consisted of fascicles of collagen (Figure 3(b)) which, with an elastic stain, were surrounded by elastic fibers 


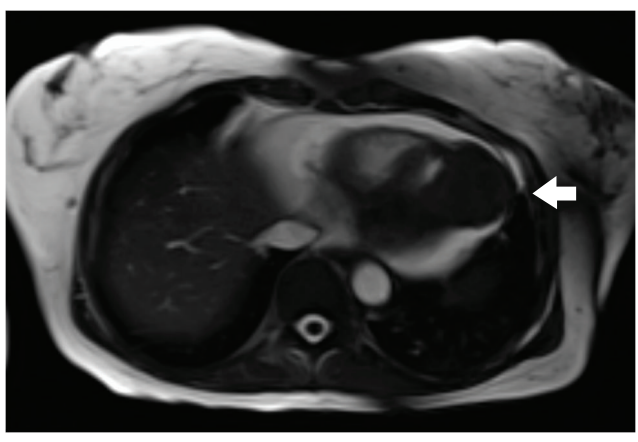

(a)

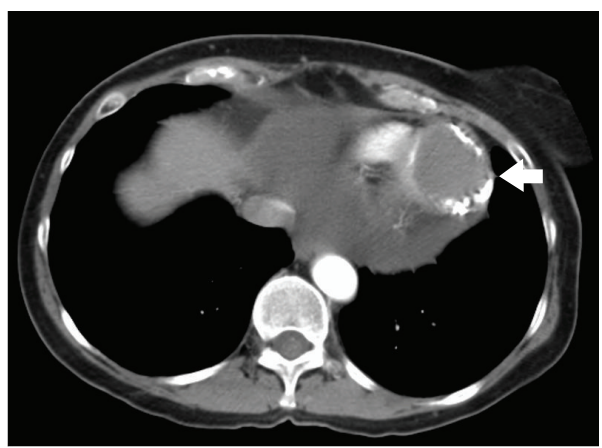

(c)

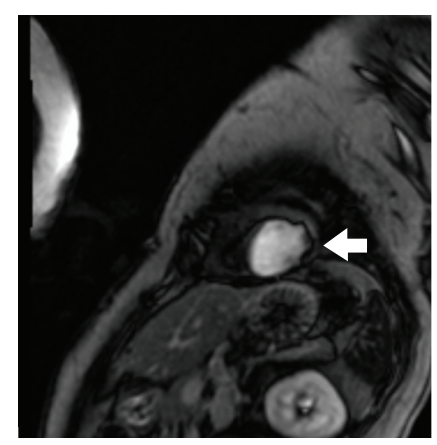

(b)

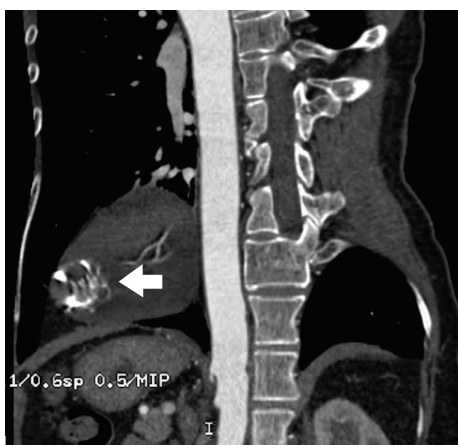

(d)

FIGURE 1: MRI imaging revealing the cardiac fibroma attached to the left ventricle (white arrow) surrounded by pericardial effusion (a and b). CT imaging revealing cardiac fibroma with peripheral calcifications (c and d).

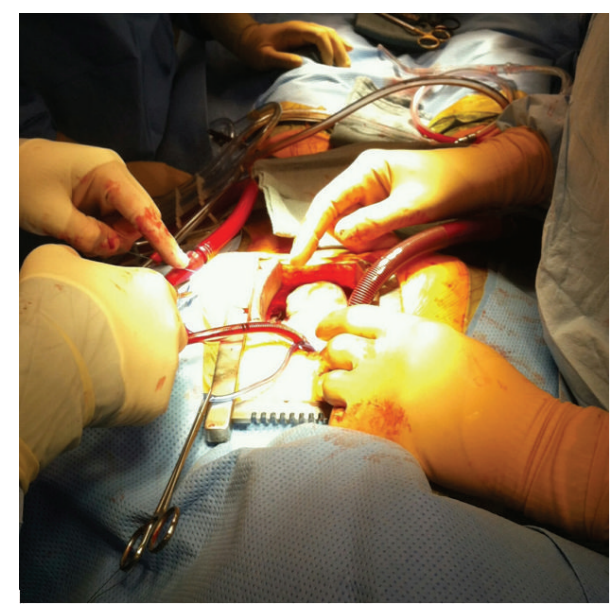

(a)

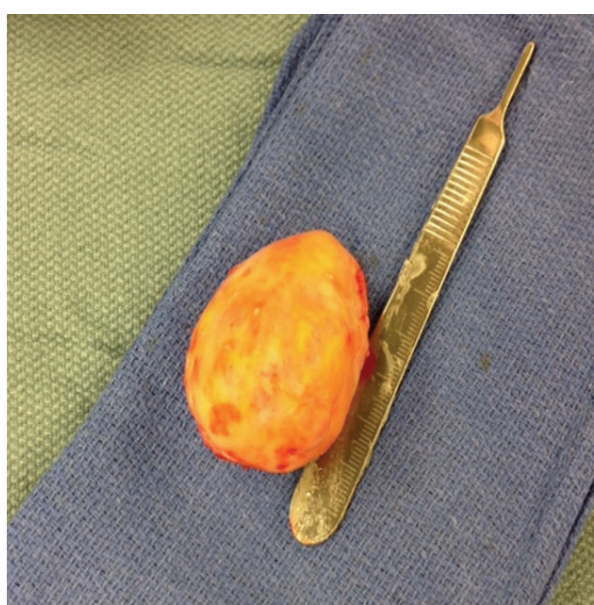

(b)

FIGURE 2: Gross image of the cardiac mass measuring $5.5 \times 4.0 \times 3.5 \mathrm{~cm}$.

(Figure 3(c)). The mass also displayed purple calcific stippling (H\&E stain) (Figure 3(d)). The patient had an uncomplicated hospital course and was discharged home on postoperative day 5.

\section{Conclusions}

This case highlights an extremely unusual finding in an adult who through various imaging modalities, surgical excision, and immunohistological analysis was found to have a cardiac fibroma in the left ventricle. While cardiac fibromas are rare solitary lesions that lack metastatic potential, they are the most common neoplastic cause of life-threatening arrhythmias and even sudden death. Largely dependent on the size and location of the tumor, patients may present with clinical manifestations relating to conduction defects, ventricular arrhythmias, congestive heart failure, and hemodynamic compromise $[3,4]$. 


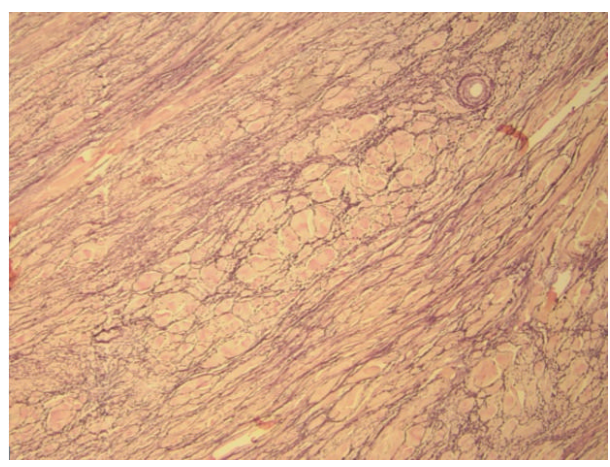

(a)

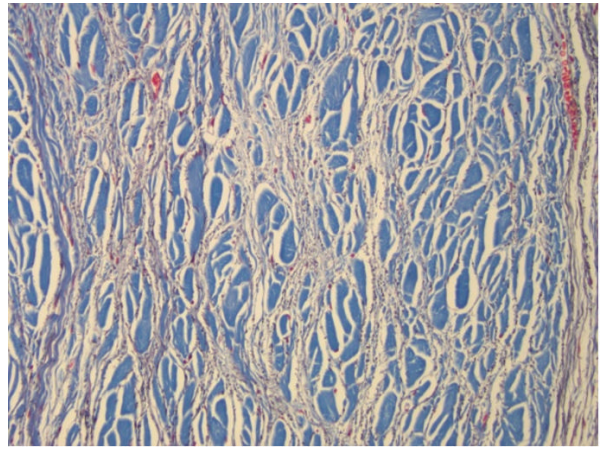

(c)

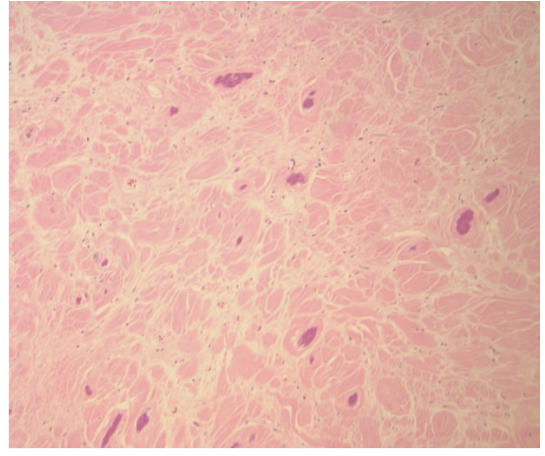

(b)

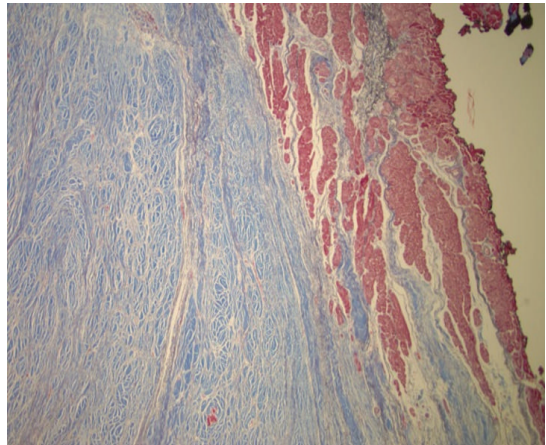

(d)

FIgURE 3: Histologic slides of the cardiac fibroma revealing numerous elastic fibers (a) and calcific stippling (b). Trichrome staining (c and d) revealed well demarcation from the interface of the tumor with the normal myocardium (red) and showed groups of fascicles of collagen (blue) surrounded by black-staining elastic fibers.

In our patient, the presenting symptoms were very nonspecific, and there were no signs of any conduction abnormalities, congestive heart failure, or hemodynamic instability. Incidental findings of a pericardial effusion and a mass on an echocardiogram prompted further investigation by cardiac MRI and CT. Surgical resection appears to be a safe and effective method for treatment.

\section{Conflict of Interests}

The authors declare that there is no conflict of interests regarding the publication of this paper.

\section{References}

[1] J. M. Cho, G. K. Danielson, F. J. Puga et al., "Surgical resection of ventricular cardiac fibromas: early and late results," Annals of Thoracic Surgery, vol. 76, no. 6, pp. 1929-1934, 2003.

[2] E. Stéphant, S. Ana, and D. Philippe, "Inter-ventricular septal cardiac fibroma in an adult: MR and MDCT features with pathologic correlation," European Journal of Radiology Extra, vol. 67, no. 3, pp. e103-e106, 2008.

[3] M. A. Padalino, C. Basso, O. Milanesi et al., "Surgically treated primary cardiac tumors in early infancy and childhood," Journal of Thoracic and Cardiovascular Surgery, vol. 129, no. 6, pp. 13581363, 2005.
[4] R. M. Freedom, K.-J. Lee, C. MacDonald, and G. Taylor, "Selected aspects of cardiac tumors in infancy and childhood," Pediatric Cardiology, vol. 21, no. 4, pp. 299-316, 2000. 


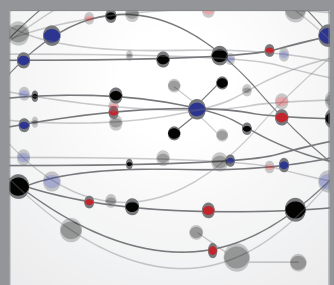

The Scientific World Journal
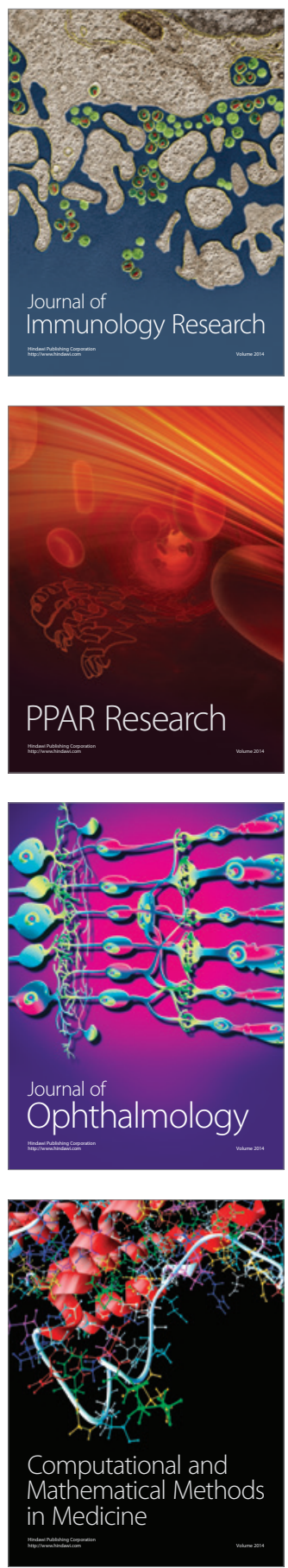

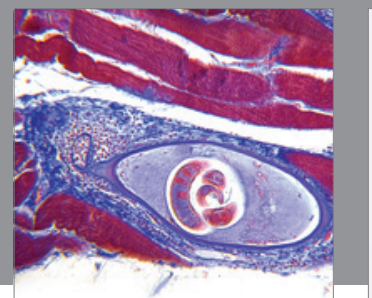

Gastroenterology

Research and Practice
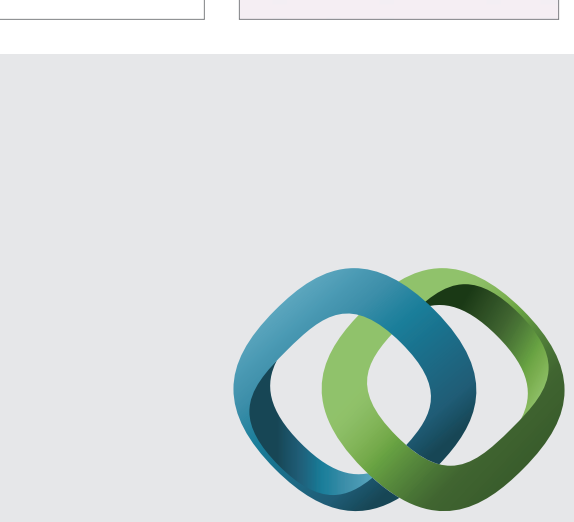

\section{Hindawi}

Submit your manuscripts at

http://www.hindawi.com
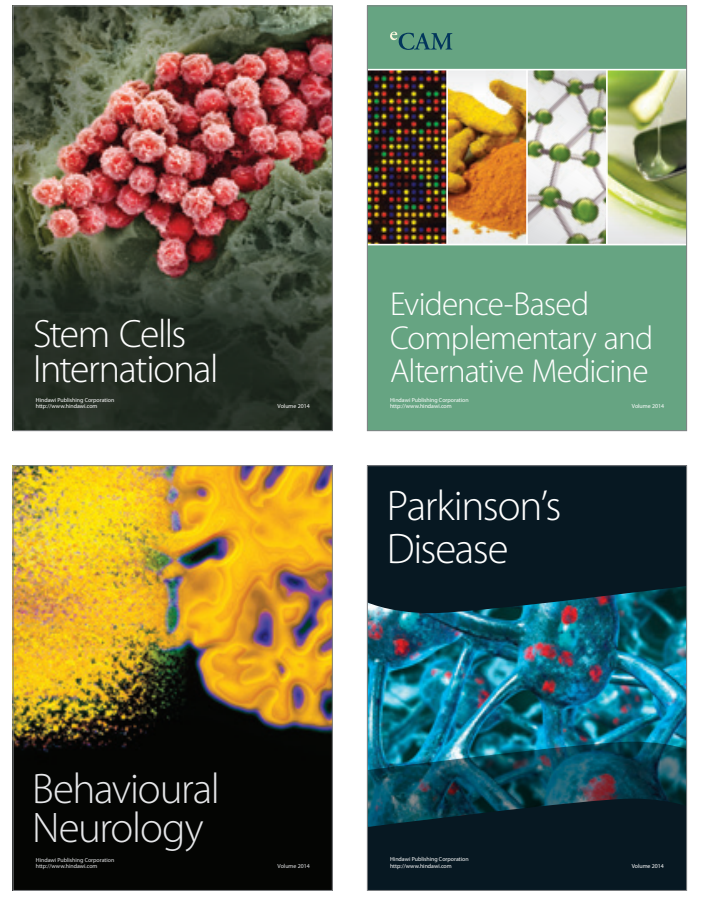
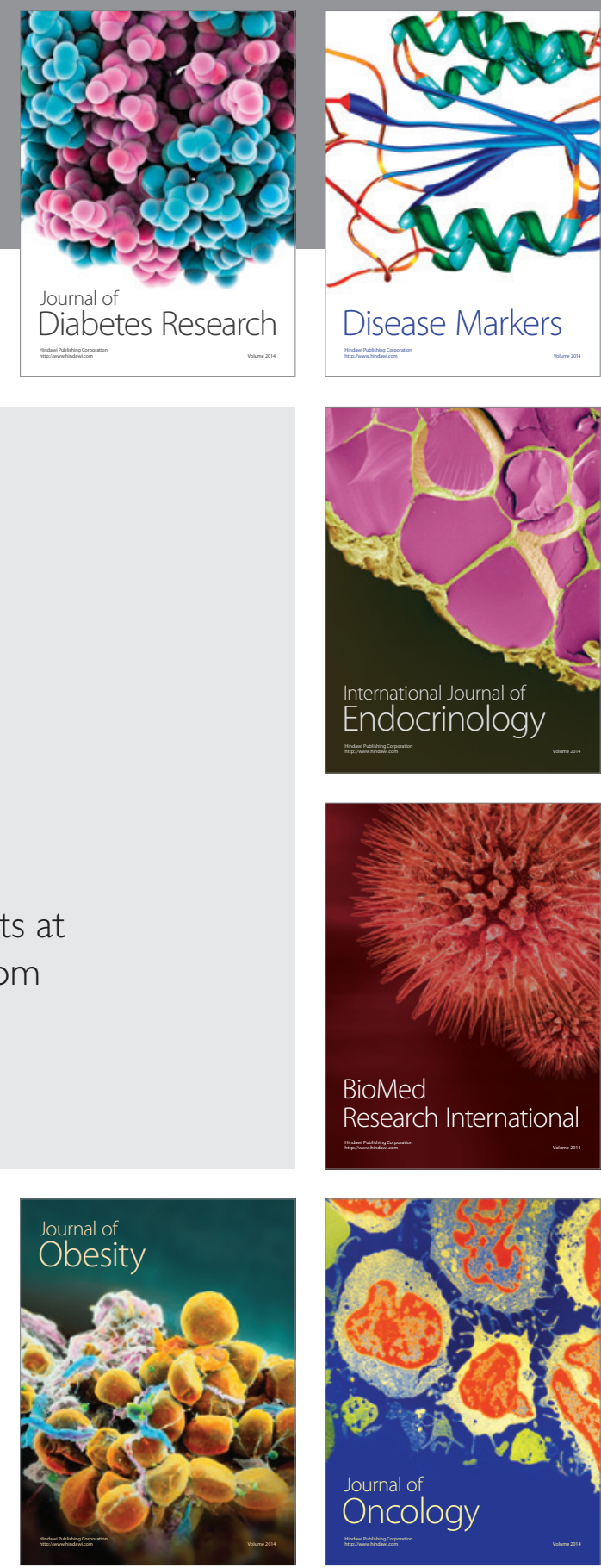

Disease Markers
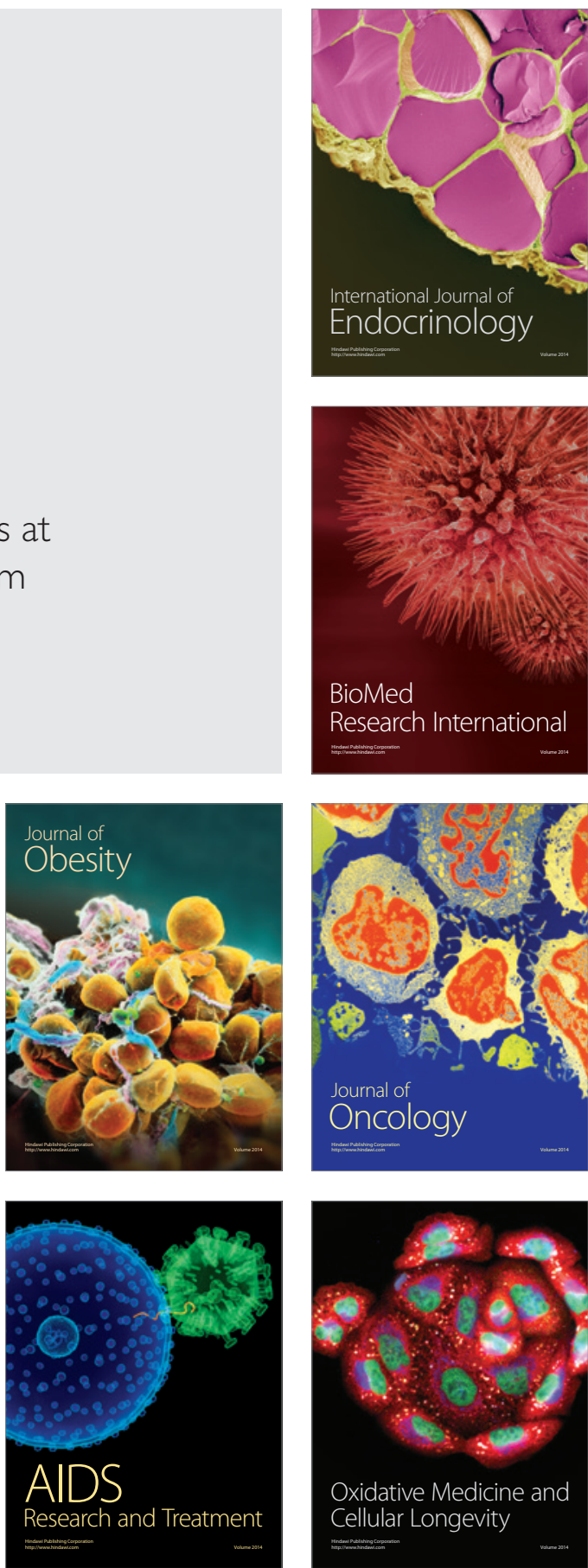